\title{
Quantitative Analysis of Calculi in 20 Cases of Canine Urolithiasis
}

\author{
Ninoska Pereira*, S.V. Upadhye, P.T. Jadhao, S.B. Akhare, \\ S.P. Salvekar and P.M. Sonkusare
}

Teaching Veterinary Clinical Complex, Nagpur Veterinary College, Nagpur, India

*Corresponding author:

\begin{tabular}{l} 
Ke y w o r d s \\
$\begin{array}{l}\text { Urolithiasis, Calculi } \\
\text { analysis, Dogs, } \\
\text { XRD }\end{array}$ \\
Article Info \\
$\begin{array}{l}\text { Accepted: } \\
\text { 04 December } 2018 \\
\text { Available Online: } \\
\text { 10 January } 2019\end{array}$ \\
\hline
\end{tabular}

A B S T R A C T

Calculi analysis to determine the chemical composition is important for treatment and prevention of urolithiasis. Quantitative crystallographic analysis using powdered X-Ray diffraction was used to study calculi of 20 cases of canine urolithiasis surgically operated between December 2017 to July 2018 at the TVCC, Nagpur Veterinary College, Nagpur. Pure phase, double phase and triple phase crystalline compounds were identified. The analysis revealed the presence of pure phases like Struvite (05 cases), Calcium carbonate (01), Calcium oxalate monohydrate (06 cases), Gabazine bromide (01 case) and combinations of Struvite and Benitoite (02 cases), Calcium oxalate monohydrate and Calcium carbonate (02 cases), Calcium carbonate and Graphite and 7- aminoheptan-2-one (02 cases), and Struvite and 1,5-penta decanediol (01 case). Amorphous material was also identified besides the mineral composition and the structural composition of the calculi were identified as orthorhombic, tetragonal and monoclinic crystals in 6 samples each, distorted orthorhombic in 5, distorted tetragonal in 3 and graphanic structure in 2 samples. Thus, the XRD was found to be very a useful method for calculi analysis in canine for advising the diet of dogs for preventing chances of recurrence.

\section{Introduction}

For effective treatment and prevention of urolithiasis, determining the chemical composition of the calculi is important. Qualitative and quantitative crystallographic analysis is useful for studying the chemical nature of uroliths. Quantitative crystallography is useful in detecting calcium oxalate, carbonate apatite, cystine, urate, and mixed uroliths (Bovee and McGuire, 1984). Optical crystallographic examination may not be adequate in determining calculi chemicals like silica, occasionally apatite and oxalate, complex salts of uric acid, and crystallized drug metabolites and residues of various types for which methods like X-ray diffraction, electron microprobe, scanning electron microscopy, infrared spectrophotometry and FTIR can be used to give more accurate urolith composition (Ruby and Ling, 1986). In view of this, the investigation was carried out to analyze the calculi using powdered X-Ray diffraction in 20 cases of canine urolithiasis surgically operated between December 2017 to July 2018 at the TVCC, Nagpur Veterinary College, Nagpur. 


\section{Materials and Methods}

The $\mathrm{X}$ ray defractometry was employed to know the mineral composition of the total 20 uroliths retrieved from the clinical cases. The XRD scan of the synthesized and subsequently sintered powder were recorded using RigakuMiniflex 600 X Ray Diffractometer with $\mathrm{CuK} \alpha$ radiation at $40 \mathrm{kV}$ voltage and 15 $\mathrm{mA}$ current supply with K-beta filter, scan speed $10.00 \mathrm{deg} / \mathrm{min}$, step width $0.0200 \mathrm{deg}$, scan range 10,000-80,000 deg. The obtained data were the compared with ICDD (International Committee for Diffraction Data) base.

\section{Results and Discussion}

The characterization of the urolith composition is important for treatment of stone related diseases and also helps in understanding the mechanism of its formation and relation to the diet and other environmental effects on dogs of different breeds and regions. The analysis of uroliths was carried out during the present investigation using powder $\mathrm{X}$-ray diffractometer (XRD). Total 20 samples were analyzed that were retrieved during the course of investigation. For characterization of the composition of the urinary calculi, powder XRD pattern of the samples was recorded. The general information regarding the quantitative analysis of the calculi and its chemical composition is given in Table 1, whereas, the Lattice information is presented in Table 2. Out of total 20 samples 13 calculi revealed 1 phase compound, 5 samples revealed 2 phase crystalline compounds and 2 samples revealed 3 phase compounds in the calculi. The analysis revealed the presence of pure phases like Struvite (05 cases), Calcium carbonate (01), Calcium oxalate monohydrate (06 cases), Gabazine bromide (01 case) and combinations of Struvite and Benitoite (02 cases), Calcium oxalate monohydrate and Calcium carbonate (02 cases), Calcium carbonate and Graphite and 7- aminoheptan-2-one (02 cases), Struvite and 1,5-penta decanediol (01 case). Besides the mineral composition, 15 samples also revealed amorphous material. Out of the total 20 calculi samples 28 , compounds were noted and the crystalline structure indicated orthorhombic, tetragonal and monoclinic crystals in 6 samples each, distorted orthorhombic in 5, distorted tetragonal in 3 and graphanic structure in 2 samples.

Bovee and McGuire (1984) observed struvite crystals as the most common calculi as also seen in the present investigation. The researchers also reported calcium oxalate, urate, silicate, cysteine and calcium phosphate calculi in dogs. During the present investigation, Struvite uroliths were observed in 8 cases. The culture tests in these cases indicated presence of microorganisms in 5 cases, thus confirming the earlier findings of Bovee and McGuire (1984).

Osborne et al.(1999) evaluated calculi composition by X-ray diffraction method and reported that struvite uroliths were the highest followed by calcium oxalate, purine, cystine, calcium phosphate, silica and mixed urolith were also identified. During the present investigation mixed urolith were observed in 7 samples. Bhatt et al. (2016) documented presence of calcium oxalate monohydrate struvite and calcium carbonate calculi, whereas mixed constituents contained calcium oxalate hydrate, struvite, whewellite and weddellite in the urinary calculi. However, Corns (1983) opined that no single method of calculi analysis was ideal and a battery of techniques should be used involving a quantitative test to get best results. According to them XRD helped in differentiating similar components in a single calculi however, it did not always help in identifying the minor components in compound composition of calculi. 
Table.1 Calculi analysis - quantitative analysis results (XRD method): General information

\begin{tabular}{|c|c|c|c|c|c|c|}
\hline $\begin{array}{l}\text { Sr } \\
\text { No }\end{array}$ & $\begin{array}{l}\text { Phase/ } \\
\text { compound name }\end{array}$ & Breed & Formula & $\begin{array}{l}\text { Nature of } \\
\text { stone }\end{array}$ & Structure & $\begin{array}{l}\text { DB ID } \\
\text { Number }\end{array}$ \\
\hline 1 & $\begin{array}{l}\mathrm{Mg}\left(\mathrm{N} \mathrm{H}_{4}\right)\left(\mathrm{P} \mathrm{O}_{4}\right) \\
\left(\mathrm{H}_{2} \mathrm{O}\right)_{6}-(\text { Struvite })\end{array}$ & Great dane & $\begin{array}{l}\mathrm{H}_{13} \mathrm{Mg}_{1} \mathrm{~N}_{1} \\
\mathrm{O}_{10} \mathrm{P}_{1}\end{array}$ & $\begin{array}{l}1 \text { Phase } \\
\text { Crystalline }\end{array}$ & Orthorhombic & 2106462 \\
\hline \multirow[t]{3}{*}{2} & $\begin{array}{l}\mathrm{Ca}\left(\mathrm{C} \mathrm{O}_{2}\right)_{2}(\mathrm{H} 2 \\
\mathrm{O})_{2} \cdot 2- \\
(\text { Calcium } \\
\text { carbonate })\end{array}$ & Pug & $\begin{array}{l}\mathrm{C}_{2} \mathrm{H}_{4} .4 \mathrm{Ca}_{1} \\
\mathrm{O}_{6} 2\end{array}$ & $\begin{array}{l}3 \text { Phase } \\
\text { Amorphus + } \\
\text { Crystalline }\end{array}$ & Tertagonal & 2310998 \\
\hline & Graphite & & $\mathrm{C}$ & & Graphanic & 9012233 \\
\hline & $\begin{array}{l}\mathrm{C}_{11} \mathrm{H}_{28} \mathrm{C}_{1} \mathrm{~N} \mathrm{O}- \\
\text { (7-aminoheptan-2- } \\
\text { one) }\end{array}$ & & $\begin{array}{l}\mathrm{C}_{11} \mathrm{H}_{28} \mathrm{C}_{1} \mathrm{~N} \\
\mathrm{O}\end{array}$ & & Monoclinic & 2004934 \\
\hline \multirow[t]{2}{*}{3} & $\begin{array}{l}\mathrm{Mg}\left(\mathrm{N} \mathrm{H}_{4}\right)\left(\mathrm{P} \mathrm{O}_{4}\right) \\
\left(\mathrm{H}_{2} \mathrm{O}\right)_{6}-(\text { struvite })\end{array}$ & $\begin{array}{l}\text { Labrador } \\
\text { retriever }\end{array}$ & $\begin{array}{l}\mathrm{H}_{13} \mathrm{Mg}_{1} \mathrm{~N}_{1} \\
\mathrm{O}_{10} \mathrm{P}_{1}\end{array}$ & $\begin{array}{l}2 \text { Phase } \\
\text { Crystalline }\end{array}$ & Orthorhombic & 2106462 \\
\hline & Benitoite & & $\mathrm{Ba} \mathrm{O}_{9} \mathrm{Si}_{3} \mathrm{Ti}$ & & $\begin{array}{l}\text { Distorted } \\
\text { Tetragonal }\end{array}$ & 9015502 \\
\hline 4 & $\begin{array}{l}\mathrm{C}_{2} \mathrm{H}_{2} \mathrm{Ca} \mathrm{O}_{5}-- \\
\text { (Calcium } \\
\text { carbonate) }\end{array}$ & Lhasa apso & $\mathrm{C}_{2} \mathrm{H}_{2} \mathrm{Ca} \mathrm{O}_{5}$ & $\begin{array}{l}1 \text { Phase } \\
\text { Crystalline }\end{array}$ & Monoclinic & 2105964 \\
\hline \multirow[t]{2}{*}{5} & $\begin{array}{l}\mathrm{Mg}\left(\mathrm{N} \mathrm{H}_{4}\right)\left(\mathrm{P} \mathrm{O}_{4}\right) \\
\left(\mathrm{H}_{2} \mathrm{O}\right)_{6}- \\
\text { (Struvite) }\end{array}$ & Pug & $\begin{array}{l}\mathrm{H}_{13} \mathrm{Mg}_{1} \mathrm{~N}_{1} \\
\mathrm{O}_{10} \mathrm{P}_{1}\end{array}$ & $\begin{array}{l}2 \text { Phase } \\
\text { Amorphus }\end{array}$ & Orthorhombic & 2106462 \\
\hline & $\begin{array}{l}1,15- \\
\text { pentadecanediol }\end{array}$ & & $\mathrm{C}_{15} \mathrm{H}_{32} \mathrm{O}_{2}$ & & Tetragonal & 2011100 \\
\hline 6 & $\begin{array}{l}\mathrm{Mg}\left(\mathrm{N} \mathrm{H}_{4}\right)\left(\mathrm{P} \mathrm{O}_{4}\right) \\
\left(\mathrm{H}_{2} \mathrm{O}\right)_{6}-(\text { Struvite })\end{array}$ & $\begin{array}{l}\text { Labrador } \\
\text { retriever }\end{array}$ & $\begin{array}{l}\mathrm{H}_{13} \mathrm{Mg}_{1} \mathrm{~N}_{1} \\
\mathrm{O}_{10} \mathrm{P}_{1}\end{array}$ & $\begin{array}{l}1 \text { Phase } \\
\text { Crystalline }\end{array}$ & Orthorhombic & 2106462 \\
\hline 7 & $\begin{array}{l}\mathrm{Ca}\left(\mathrm{C} \mathrm{O}_{2}\right)_{2}\left(\mathrm{H}_{2}\right. \\
\mathrm{O})_{2} .2-(\text { Calcium } \\
\text { carbonate })\end{array}$ & Mongrel & $\begin{array}{l}\mathrm{C}_{2} \mathrm{H}_{4} \cdot 4 \mathrm{Ca}_{1} \\
\mathrm{O}_{6} 2\end{array}$ & $\begin{array}{l}1 \text { Phase } \\
\text { Crystalline + } \\
\text { Amorphous }\end{array}$ & Tetragonal & 2310998 \\
\hline 8 & $\begin{array}{l}\mathrm{MRHBrH}_{2} \mathrm{O}- \\
\text { (Gabazine } \\
\text { bromide) }\end{array}$ & Dalmatian & $\begin{array}{l}\mathrm{C}_{15} \mathrm{H}_{18} \mathrm{Br} \mathrm{N} \mathrm{N}_{3} \\
\mathrm{O}_{3}\end{array}$ & $\begin{array}{l}1 \text { Phase } \\
\text { Crystalline + } \\
\text { Amorphous }\end{array}$ & $\begin{array}{l}\text { Distorted } \\
\text { orthorhombic }\end{array}$ & 4101243 \\
\hline \multirow[t]{2}{*}{9} & $\begin{array}{l}\mathrm{C}_{2} \mathrm{H}_{2} \mathrm{Ca} \mathrm{O}_{5}- \\
\text { (Calcium oxalate } \\
\text { monohydrate) }\end{array}$ & Pug & $\mathrm{C}_{2} \mathrm{H}_{2} \mathrm{Ca} \mathrm{O}_{5}$ & $\begin{array}{l}2 \text { Phase } \\
\text { Crystalline }+ \\
\text { Few } \\
\text { Amorphous }\end{array}$ & Monoclinic & 2105964 \\
\hline & $\begin{array}{l}\mathrm{Ca}\left(\mathrm{C} \mathrm{O}_{2}\right)_{2}\left(\mathrm{H}_{2}\right. \\
\mathrm{O})_{2} .2-(\text { Calcium } \\
\text { Carbonate })\end{array}$ & & $\begin{array}{l}\mathrm{C}_{2} \mathrm{H}_{4} \cdot 4 \mathrm{Ca}_{1} \\
\mathrm{O}_{6} 2\end{array}$ & & Tetragonal & 2310998 \\
\hline
\end{tabular}


Table.1 (Contd....): Calculi analysis -Quantitative analysis results (XRD method): General information

\begin{tabular}{|c|c|c|c|c|c|c|}
\hline $\begin{array}{l}\text { Sr } \\
\text { No }\end{array}$ & $\begin{array}{l}\text { Phase/ compound } \\
\text { name }\end{array}$ & Breed & Formula & $\begin{array}{l}\text { Nature of } \\
\text { stone }\end{array}$ & Structure & $\begin{array}{l}\text { DB ID } \\
\text { Number }\end{array}$ \\
\hline 10 & $\begin{array}{l}\mathrm{C}_{2} \mathrm{H}_{2} \mathrm{Ca} \mathrm{O}_{5}- \\
\text { (Calcium oxalate } \\
\text { monohydrate) }\end{array}$ & Pug & $\begin{array}{l}\mathrm{C}_{2} \mathrm{H}_{2} \mathrm{Ca} \\
\mathrm{O}_{5}\end{array}$ & $\begin{array}{l}1 \text { Phase } \\
\text { Crystalline + } \\
\text { Amorphus }\end{array}$ & Monoclinic & 2105964 \\
\hline 11 & $\begin{array}{l}\mathrm{C}_{2} \mathrm{H}_{2} \mathrm{Ca} \mathrm{O}_{5}- \\
\text { (Calcium oxalate } \\
\text { monohydrate ) }\end{array}$ & Spitz & $\begin{array}{l}\mathrm{C}_{2} \mathrm{H}_{2} \mathrm{Ca} \\
\mathrm{O}_{5}\end{array}$ & $\begin{array}{l}1 \text { Phase } \\
\text { Crystalline + } \\
\text { Amorphus }\end{array}$ & Monoclinic & 2105964 \\
\hline 12 & $\begin{array}{l}\mathrm{Mg}\left(\mathrm{N} \mathrm{H}_{4}\right)\left(\mathrm{P} \mathrm{O}_{4}\right)\left(\mathrm{H}_{2}\right. \\
\mathrm{O})_{6}-(\text { struvite })\end{array}$ & $\begin{array}{l}\text { Labrador } \\
\text { retriever }\end{array}$ & $\begin{array}{l}\mathrm{H}_{13} \mathrm{Mg}_{1} \\
\mathrm{~N}_{1} \mathrm{O}_{10} \mathrm{P}_{1}\end{array}$ & $\begin{array}{l}1 \text { Phase } \\
\text { Crystalline + } \\
\text { Amorphus }\end{array}$ & Orthorhombic & 2106462 \\
\hline \multirow[t]{2}{*}{13} & $\begin{array}{l}\mathrm{C}_{2} \mathrm{H}_{2} \mathrm{Ca} \mathrm{O}_{5}- \\
\text { (Calcium oxalate } \\
\text { monohydrate ) }\end{array}$ & Mongrel & $\begin{array}{l}\mathrm{C}_{2} \mathrm{H}_{2} \mathrm{Ca} \\
\mathrm{O}_{5}\end{array}$ & $\begin{array}{l}2 \text { Phase } \\
\text { Crystalline + } \\
\text { Amorphus }\end{array}$ & Monoclinic & 2105964 \\
\hline & $\begin{array}{l}\mathrm{Ca}\left(\mathrm{C} \mathrm{O}_{2}\right)_{2}\left(\mathrm{H}_{2} \mathrm{O}\right)_{2} .2- \\
\text { (Calcium carbonate) }\end{array}$ & & $\begin{array}{l}\mathrm{C}_{2} \mathrm{H}_{4} \cdot 4 \\
\mathrm{Ca}_{1} \mathrm{O}_{6} 2\end{array}$ & & Tertagonal & 2310998 \\
\hline 14 & $\begin{array}{l}\mathrm{C}_{2} \mathrm{H}_{2} \mathrm{Ca} \mathrm{O}_{5} \text { - } \\
\text { (Calcium oxalate } \\
\text { monohydrate) }\end{array}$ & Pug & $\begin{array}{l}\mathrm{C}_{2} \mathrm{H}_{2} \mathrm{Ca} \\
\mathrm{O}_{5}\end{array}$ & $\begin{array}{l}1 \text { Phase } \\
\text { Crystalline + } \\
\text { Amorphus }\end{array}$ & $\begin{array}{l}\text { Distorted } \\
\text { orthorhombic }\end{array}$ & 2105964 \\
\hline 15 & $\begin{array}{l}\mathrm{C}_{2} \mathrm{H}_{2} \mathrm{Ca} \mathrm{O}_{5}- \\
\text { (Calcium oxalate } \\
\text { monohydrate ) }\end{array}$ & Mongrel & $\begin{array}{l}\mathrm{C}_{2} \mathrm{H}_{2} \mathrm{Ca} \\
\mathrm{O}_{5}\end{array}$ & $\begin{array}{l}1 \text { Phase } \\
\text { Crystalline + } \\
\text { Amorphus }\end{array}$ & $\begin{array}{l}\text { Distorted } \\
\text { orthorhombic }\end{array}$ & 2105964 \\
\hline 16 & $\begin{array}{l}\mathrm{Mg}\left(\mathrm{N} \mathrm{H}_{4}\right)\left(\mathrm{P} \mathrm{O}_{4}\right)\left(\mathrm{H}_{2}\right. \\
\mathrm{O})_{6}-(\text { Struvite })\end{array}$ & Pug & $\begin{array}{l}\mathrm{H}_{13} \mathrm{Mg}_{1} \\
\mathrm{~N}_{1} \mathrm{O}_{10} \mathrm{P}_{1}\end{array}$ & $\begin{array}{l}1 \text { Phase } \\
\text { Crystalline }\end{array}$ & $\begin{array}{l}\text { Distorted } \\
\text { orthorhombic }\end{array}$ & 2106462 \\
\hline 17 & $\begin{array}{l}\mathrm{C}_{2} \mathrm{H}_{2} \mathrm{Ca} \mathrm{O}_{5}- \\
\text { (Calcium oxalate } \\
\text { monohydrate) }\end{array}$ & Mongrel & $\begin{array}{l}\mathrm{C}_{2} \mathrm{H}_{2} \mathrm{Ca} \\
\mathrm{O}_{5}\end{array}$ & $\begin{array}{l}1 \text { Phase } \\
\text { Crystalline + } \\
\text { Amorphus }\end{array}$ & $\begin{array}{l}\text { Distorted } \\
\text { orthorhombic }\end{array}$ & 2105964 \\
\hline \multirow[t]{3}{*}{18} & $\begin{array}{l}\mathrm{Ca}\left(\mathrm{C} \mathrm{O}_{2}\right)_{2}\left(\mathrm{H}_{2} \mathrm{O}\right)_{2} .2- \\
\text { (calcium carbonate) }\end{array}$ & Pug & $\begin{array}{l}\mathrm{C}_{2} \mathrm{H}_{4} \cdot 4 \\
\mathrm{Ca}_{1} \mathrm{O}_{6} 2\end{array}$ & $\begin{array}{l}3 \text { Phase } \\
\text { Crystalline + } \\
\text { Amorphus }\end{array}$ & Tetragonal & 2310998 \\
\hline & Graphite & & $\mathrm{C}$ & & Graphanic & 9012233 \\
\hline & $\begin{array}{l}\mathrm{C}_{11} \mathrm{H}_{28} \mathrm{Cl} \mathrm{N} \mathrm{O}-(7- \\
\text { aminoheptan-2-one })\end{array}$ & & $\begin{array}{l}\mathrm{C} 11 \mathrm{H} 28 \\
\mathrm{ClNO}\end{array}$ & & $\begin{array}{l}\text { Distorted } \\
\text { Tertagonal }\end{array}$ & 2004934 \\
\hline \multirow[t]{2}{*}{19} & $\begin{array}{l}\mathrm{Mg}\left(\mathrm{N} \mathrm{H}_{4}\right)\left(\mathrm{P} \mathrm{O}_{4}\right)\left(\mathrm{H}_{2}\right. \\
\mathrm{O})_{6}-(\text { Struvite })\end{array}$ & Labrador & $\begin{array}{l}\mathrm{H}_{13} \mathrm{Mg}_{1} \\
\mathrm{~N}_{1} \mathrm{O}_{10} \mathrm{P}_{1}\end{array}$ & $\begin{array}{l}2 \text { Phase } \\
\text { Crystalline }\end{array}$ & Orthorhombic & 2106462 \\
\hline & Benitoite & & $\begin{array}{l}\mathrm{Ba} \mathrm{O}_{9} \mathrm{Si}_{3} \\
\mathrm{Ti}\end{array}$ & & $\begin{array}{l}\text { Distorted } \\
\text { Tetragonal }\end{array}$ & 9015502 \\
\hline 20 & $\begin{array}{l}\mathrm{Mg}\left(\mathrm{N} \mathrm{H}_{4}\right)\left(\mathrm{P} \mathrm{O}_{4}\right)\left(\mathrm{H}_{2}\right. \\
\mathrm{O})_{6}-(\text { Struvite })\end{array}$ & $\begin{array}{l}\text { Lhasa } \\
\text { apso }\end{array}$ & $\begin{array}{l}\mathrm{H}_{13} \mathrm{Mg}_{1} \\
\mathrm{~N}_{1} \mathrm{O}_{10} \mathrm{P}_{1}\end{array}$ & $\begin{array}{l}1 \text { Phase } \\
\text { Crystalline }\end{array}$ & Orthorhombic & 2106462 \\
\hline
\end{tabular}


Table.2 Calculi analysis -Quantitative analysis results (XRD method): Lattice information

\begin{tabular}{|c|c|c|c|c|c|c|c|c|c|c|}
\hline Sr No & Breed & Phase/ compound name & $\mathrm{a}(\mathrm{A})$ & $\mathrm{b}(\mathrm{A})$ & $\mathrm{c}(\mathrm{A})$ & $\begin{array}{l}\text { alpha } \\
\text { (deg) }\end{array}$ & beta (deg) & $\begin{array}{l}\text { gamma } \\
\text { (deg) }\end{array}$ & $\mathrm{V}\left(\mathrm{A}^{\wedge} 3\right)$ & Space group \\
\hline 1 & Great Dane & $\begin{array}{l}\mathrm{Mg}\left(\mathrm{N} \mathrm{H}_{4}\right)\left(\mathrm{P} \mathrm{O}_{4}\right)\left(\mathrm{H}_{2} \mathrm{O}\right)_{6} \\
-(\text { Struvite })\end{array}$ & $\begin{array}{l}6.9456 \\
39\end{array}$ & 6.136024 & 11.20616 & 90 & 90 & 90 & 477.590795 & $31:$ Pmn 21 \\
\hline \multirow[t]{3}{*}{2} & Pug & $\begin{array}{l}\mathrm{Ca}\left(\mathrm{C} \mathrm{O}_{2}\right)_{2}(\mathrm{H} 2 \mathrm{O})_{2} .2- \\
\text { (Calcium carbonate) }\end{array}$ & 12.39777 & 12.39777 & 7.367685 & 90 & 90 & 90 & 1132.447556 & $87: \mathrm{I} 4 / \mathrm{m}$ \\
\hline & & Graphite & 4.703251 & 5.956181 & 4.427659 & 90 & 90 & 90 & 124.03384 & 54 : Pcca \\
\hline & & $\begin{array}{l}\mathrm{C}_{11} \mathrm{H}_{28} \mathrm{C}_{1} \mathrm{~N} \mathrm{O}- \\
\text { (7-aminoheptan-2-one) }\end{array}$ & 7.735467 & 40.19912 & 4.664484 & 90 & 107.339996 & 90 & 1384.54376 & $\begin{array}{l}14: \\
\text { P121/a1,unique- } \\
\text { b,cell-3 }\end{array}$ \\
\hline \multirow[t]{2}{*}{3} & $\begin{array}{l}\text { Labrador } \\
\text { Retriever }\end{array}$ & $\begin{array}{l}\mathrm{Mg}\left(\mathrm{N} \mathrm{H}_{4}\right)\left(\mathrm{P} \mathrm{O}_{4}\right)\left(\mathrm{H}_{2} \mathrm{O}\right)_{6} \\
\text { - (struvite) }\end{array}$ & 6.942781 & 6.137159 & 11.19282 & 90 & 90 & 90 & 476.914297 & $31:$ Pmn 21 \\
\hline & & Benitoite & 11.24678 & 11.24678 & 9.568958 & 90 & 90 & 120 & 1048.218775 & $159: \mathrm{P} 31 \mathrm{c}$ \\
\hline 4 & Lasa Apso & $\begin{array}{l}\mathrm{C}_{2} \mathrm{H}_{2} \mathrm{Ca} \mathrm{O}_{5} \text { _-(Calcium } \\
\text { carbonate) }\end{array}$ & 9.987673 & 7.302072 & 6.2981 & 90 & 107.07 & 90 & 439.090153 & $\begin{array}{l}12: \\
\text { I1 } 2 / \mathrm{m} 1 \text {, unique-b, } \\
\text { cell-3 }\end{array}$ \\
\hline \multirow[t]{2}{*}{5} & Pug & $\begin{array}{l}\mathrm{Mg}\left(\mathrm{N} \mathrm{H}_{4}\right)\left(\mathrm{P} \mathrm{O}_{4}\right)\left(\mathrm{H}_{2} \mathrm{O}\right)_{6} \\
\text { - (Struvite) }\end{array}$ & 6.957194 & 6.141543 & 11.22027 & 90 & 90 & 90 & 479.418801 & $31:$ Pmn21 \\
\hline & & 1,15-pentadecanediol & 7.310404 & 42.61065 & 5.063505 & 90 & 90 & 90 & 1577.286989 & 19 : P212121 \\
\hline 6 & $\begin{array}{l}\text { Labrador } \\
\text { Retriever }\end{array}$ & $\begin{array}{l}\mathrm{Mg}\left(\mathrm{N} \mathrm{H}_{4}\right)\left(\mathrm{P} \mathrm{O}_{4}\right)\left(\mathrm{H}_{2} \mathrm{O}\right)_{6} \\
\text { - (Struvite) }\end{array}$ & 6.950281 & 6.144647 & 11.21288 & 90 & 90 & 90 & 478.868483 & $31:$ Pmn21 \\
\hline 7 & Mongrel & $\begin{array}{l}\mathrm{Ca}\left(\mathrm{C} \mathrm{O}_{2}\right)_{2}\left(\mathrm{H}_{2} \mathrm{O}\right)_{2} .2- \\
\text { (Calcium carbonate) }\end{array}$ & 12.37651 & 12.37651 & 7.355816 & 90 & 90 & 90 & 1126.749295 & $87: I 4 / m$ \\
\hline 8 & Dalmatian & $\begin{array}{l}\mathrm{MRHBrH}_{2} \mathrm{O}-(\text { Gabazine } \\
\text { bromide })\end{array}$ & 7.870705 & 11.8689 & 16.62125 & 90 & 92.568001 & 90 & 1551.142194 & $\begin{array}{l}14: \\
\text { P121/c1, unique- } \\
\text { b,cell-1 }\end{array}$ \\
\hline \multirow[t]{2}{*}{9} & Pug & $\begin{array}{l}\mathrm{C}_{2} \mathrm{H}_{2} \mathrm{Ca} \mathrm{O}_{5} \text {-(Calcium } \\
\text { oxalate monohydrate })\end{array}$ & 10.00382 & 7.313878 & 6.308282 & 90 & 107.07 & 90 & 441.223327 & $\begin{array}{l}12: \\
\text { I1 } 2 / \mathrm{m} 1 \text {, unique- } \\
\mathrm{b}, \text { cell-3 }\end{array}$ \\
\hline & & $\begin{array}{l}\mathrm{Ca}\left(\mathrm{C} \mathrm{O}_{2}\right)_{2}\left(\mathrm{H}_{2} \mathrm{O}\right)_{2} .2- \\
\text { (Calcium Carbonate) }\end{array}$ & 12.41292 & 12.41292 & 7.361348 & 90 & 90 & 90 & 1134.240822 & $87: I 4 / m$ \\
\hline 10 & Pug & $\begin{array}{l}\mathrm{C}_{2} \mathrm{H}_{2} \mathrm{Ca} \mathrm{O}_{5}-(\text { Calcium } \\
\text { oxalate monohydrate) }\end{array}$ & 9.985276 & 7.30032 & 6.296588 & 90 & 107.07 & 90 & 438.774114 & $\begin{array}{l}12: \\
\mathrm{I} 12 / \mathrm{m} 1 \text {, unique- }\end{array}$ \\
\hline
\end{tabular}


Int.J.Curr.Microbiol.App.Sci (2019) 8(1): 314-321

\begin{tabular}{|c|c|c|c|c|c|c|c|c|c|c|}
\hline & & & & & & & & & & b,cell-3 \\
\hline 11 & Spitz & $\begin{array}{l}\mathrm{C}_{2} \mathrm{H}_{2} \mathrm{Ca} \mathrm{O}_{5}-(\text { Calcium } \\
\text { oxalate monohydrate ) }\end{array}$ & 9.98327 & 7.298853 & 6.295323 & 90 & 107.07 & 90 & 438.509703 & $\begin{array}{l}12: \\
\text { I1 } 2 / m 1 \text {, unique- } \\
\text { b,cell-3 }\end{array}$ \\
\hline 12 & $\begin{array}{l}\text { Labrador } \\
\text { retriever }\end{array}$ & $\begin{array}{l}\mathrm{Mg}\left(\mathrm{N} \mathrm{H}_{4}\right)\left(\mathrm{P} \mathrm{O}_{4}\right)\left(\mathrm{H}_{2} \mathrm{O}\right)_{6} \\
\text { (struvite) }\end{array}$ & 6.953131 & 6.133929 & 11.20025 & 90 & 90 & 90 & 477.690591 & $31:$ Pmn21 \\
\hline \multirow[t]{2}{*}{13} & Mongrel & $\begin{array}{l}\mathrm{C}_{2} \mathrm{H}_{2} \mathrm{Ca} \mathrm{O}_{5}-(\text { Calcium } \\
\text { oxalate monohydrate })\end{array}$ & 9.997383 & 7.309172 & 6.304223 & 90 & 107.07 & 90 & 440.372108 & $\begin{array}{l}12: \\
\text { I12/m1,unique- } \\
\mathrm{b}, \text { cell-3 }\end{array}$ \\
\hline & & $\begin{array}{l}\mathrm{Ca}\left(\mathrm{C} \mathrm{O}_{2}\right)_{2}\left(\mathrm{H}_{2} \mathrm{O}\right)_{2} .2- \\
\text { (Calcium carbonate) }\end{array}$ & 12.38554 & 12.38554 & 7.362706 & 90 & 90 & 90 & 1129.451074 & $87: I 4 / m$ \\
\hline 14 & Pug & $\begin{array}{l}\mathrm{C}_{2} \mathrm{H}_{2} \mathrm{Ca} \mathrm{O}_{5} \text {-( Calcium } \\
\text { oxalate monohydrate) }\end{array}$ & 9.990467 & 7.304115 & 6.299861 & 90 & 107.07 & 90 & 439.458747 & $\begin{array}{l}12: \\
\mathrm{I} 12 / \mathrm{m} 1 \text {, unique- } \\
\mathrm{b}, \text { cell-3 }\end{array}$ \\
\hline 15 & Mongrel & $\begin{array}{l}\mathrm{C}_{2} \mathrm{H}_{2} \mathrm{Ca} \mathrm{O}_{5}-(\text { Calcium } \\
\text { oxalate monohydrate })\end{array}$ & $\begin{array}{l}\mathrm{C} 2 \mathrm{H} 2 \\
\mathrm{Ca} \text { O5 }\end{array}$ & 9.982843 & 7.298541 & $\begin{array}{l}6.295 \\
054\end{array}$ & 90 & 107.07 & 90 & $\begin{array}{l}12: \\
\text { I1 } 2 / \mathrm{m} 1 \text {,unique- } \\
\text { b,cell-3 }\end{array}$ \\
\hline 16 & Pug & $\begin{array}{l}\mathrm{Mg}\left(\mathrm{N} \mathrm{H}_{4}\right)\left(\mathrm{P} \mathrm{O}_{4}\right)\left(\mathrm{H}_{2} \mathrm{O}\right)_{6} \\
\text { - (Struvite) }\end{array}$ & 6.945639 & 6.136024 & 11.20616 & 90 & 90 & 90 & 477.590795 & $31:$ Pmn21 \\
\hline 17 & Mongrel & $\begin{array}{l}\mathrm{C}_{2} \mathrm{H}_{2} \mathrm{Ca} \mathrm{O}_{5}-(\text { Calcium } \\
\text { oxalate monohydrate })\end{array}$ & 9.987673 & 7.302072 & 6.2981 & 90 & 107.07 & 90 & 439.090153 & $\begin{array}{l}12: \\
\text { I1 } 2 / \mathrm{m} 1 \text {,unique- } \\
\mathrm{b}, \text { cell-3 }\end{array}$ \\
\hline \multirow[t]{3}{*}{18} & Pug & $\begin{array}{l}\mathrm{Ca}\left(\mathrm{C} \mathrm{O}_{2}\right)_{2}\left(\mathrm{H}_{2} \mathrm{O}\right)_{2} .2- \\
\text { (calcium carbonate) }\end{array}$ & 12.39777 & 12.39777 & 7.367685 & 90 & 90 & 90 & 1132.447556 & $87: \mathrm{I} 4 / \mathrm{m}$ \\
\hline & & Graphite & 4.703251 & 5.956181 & 4.427659 & 90 & 90 & 90 & 124.03384 & $54:$ Pcca \\
\hline & & $\begin{array}{l}\mathrm{C}_{11} \mathrm{H}_{28} \mathrm{Cl} \mathrm{N} \mathrm{O} \text {-( 7- } \\
\text { aminoheptan-2-one) }\end{array}$ & 7.735467 & 40.19912 & 4.664484 & 90 & 107.339996 & 90 & 1384.54376 & $\begin{array}{l}14: \\
\text { P121/a1, unique- } \\
\text { b,cell-3 }\end{array}$ \\
\hline \multirow[t]{2}{*}{19} & Labrador & $\begin{array}{l}\mathrm{Mg}\left(\mathrm{N} \mathrm{H}_{4}\right)\left(\mathrm{P} \mathrm{O}_{4}\right)\left(\mathrm{H}_{2} \mathrm{O}\right)_{6} \\
\text { - (Struvite) }\end{array}$ & 6.942781 & 6.137159 & 11.19282 & 90 & 90 & 90 & 476.914297 & $31:$ Pmn21 \\
\hline & & Benitoite & 11.24678 & 11.24678 & 9.568958 & 90 & 90 & 120 & 1048.218775 & 159 : P31c \\
\hline 20 & Lhasa apso & $\begin{array}{l}\mathrm{Mg}\left(\mathrm{N} \mathrm{H}_{4}\right)\left(\mathrm{P} \mathrm{O}_{4}\right)\left(\mathrm{H}_{2}\right. \\
\mathrm{O})_{6}-(\text { Struvite })\end{array}$ & 6.950281 & 6.144647 & 11.21288 & 90 & 90 & 90 & 478.868483 & $31:$ Pmn21 \\
\hline
\end{tabular}


Int.J.Curr.Microbiol.App.Sci (2019) 8(1): 314-321

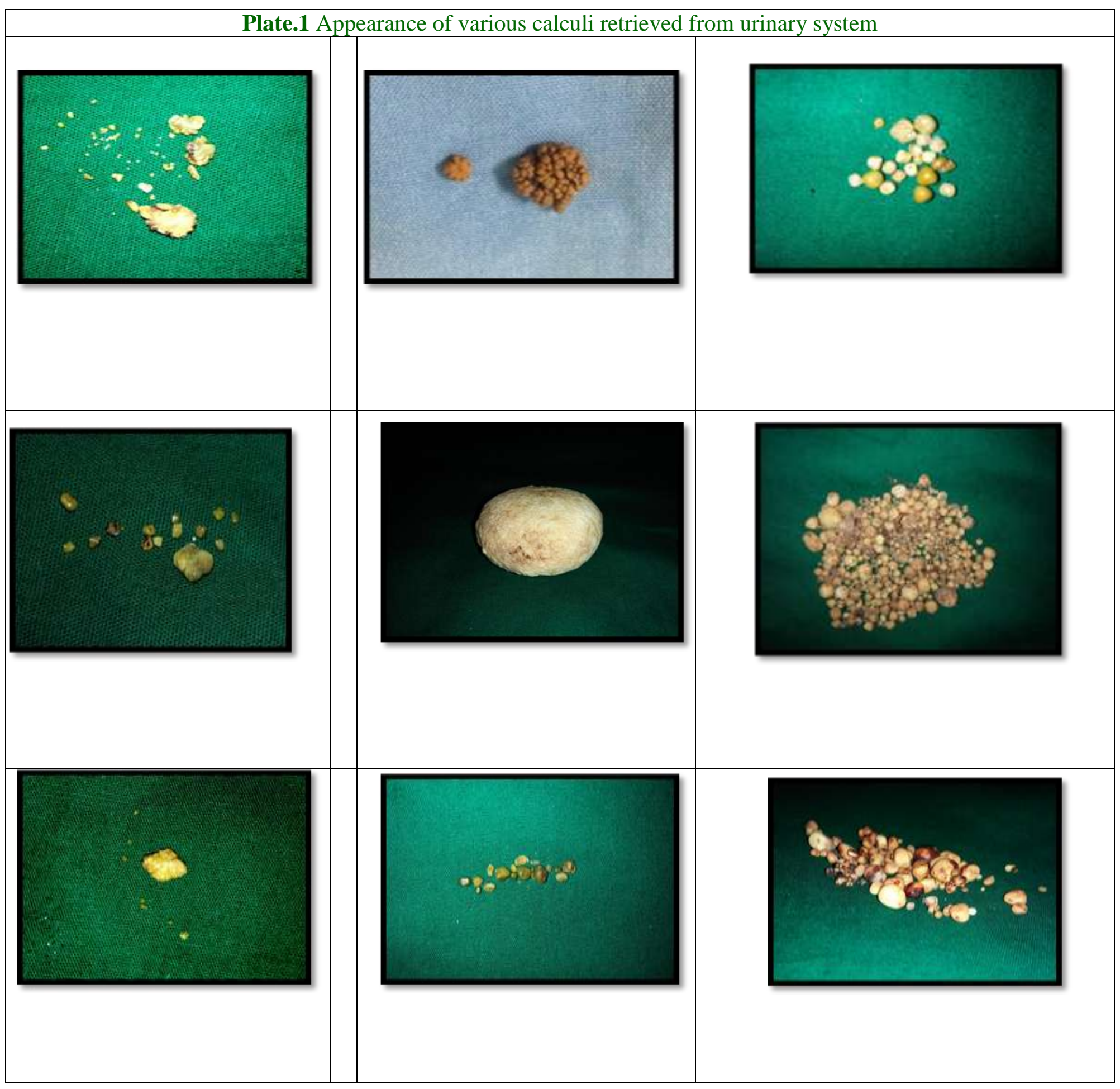


In conclusion x-ray diffractometry revealed struvite calculi ( $40 \%$ ) to be the most common followed by calcium carbonate (25\%), calcium oxalate monohydrate $(10 \%)$, 7-aminoheptan-2-one (10\%), benetoite (10\%), graphite $(10 \%)$, gabazine bromide $(5 \%)$ and 1,5-pentadecanediol (5\%. Out of 20 samples 13 calculi revealed single phase, 5 samples double phase and 2 samples had 3 phase compounds in the calculi.

\section{References}

Bhatt, P.A., R.P. Laiya, D.B. Patil, P.V. Parikh and P. Paul (2016) Analysis of the constituents of urinary calculi of dog using various scientific techniques. Current Science, 110(5): 863-867.

Bovee, K.C. and T. McGuire (1984) Qualitative and Quantitative analysis of uroliths in dogs: Definitive determination of chemical type. J.Am.Vet.Med.Assoc., 185(9): 983987.

Corns, C.M. (1983) Infrared analysis of renal calculi: a comparison with conventional techniques. Ann. Clin. Biochem. 20:20-25.

Osborne, C.A., J.P. Lulich, J.W. Bartges, L.A. Koehler, L.K. Urlich, K.A. Bird and L.L. Swanson (1999). Medical dissolution and prevention of canine struvite urolithiasis. Veterinary Clinics of North America: Small animal Practice 29: 73-111.

Ruby, A.L., and Ling, G.V (1986) Methods of Analysis of Canine Uroliths. Veterinary Clinics of North America: Small Animal Practice. 16(2): 293301.

\section{How to cite this article:}

Ninoska Pereira, S.V. Upadhye, P.T. Jadhao, S.B. Akhare, S.P. Salvekar and Sonkusare, P.M. 2019. Quantitative Analysis of Calculi in 20 Cases of Canine Urolithiasis. Int.J.Curr.Microbiol.App.Sci. 8(01): 314-321. doi: https://doi.org/10.20546/ijcmas.2019.801.033 